\title{
Convex Geometry and Mathematical Morphology in a Generalized Structure
}

\author{
Ramkumar P.B \\ Rajagiri School of Engineering \& \\ Technology \\ Rajagiri Valley \\ Cochin
}

\author{
Pramod K.V \\ Cochin University of Science \& \\ Technology \\ Department of Computer \\ Applications, Cochin
}

\begin{abstract}
The aim of this paper is to find relationship between Poset Geometry and Mathematical Morphology. We characterize morphological convex geometries, using the definition of Moore family. We also show that this can be connected to Alexandroff space. We also presented an algebraic framework for Mathematical Morphology .
\end{abstract}

\section{General Terms}

Mathematical Morphology, Image Processing..

\section{Keywords}

Poset Geometry, Moore family, Alexandroff space, Dilation, Erosion, Morphological closure, convex geometry ,Morphological space.

\section{INTRODUCTION}

Mathematical Morphology originally developed in 1967 by Georges Matheron[14] and Jean Serra[13] at the Paris School of Mines. Mathematical morphology is based on mathematical concepts from set theory and uses a number of operators that are useful for edge detection, noise removal, image enhancement, and image segmentation etc.

The two basic operations of mathematical morphology are erosion and dilation. These operators use an input image to be eroded or dilated and a structuring element. In convex analysis and optimization the nonlinear signal operation $\oplus$ is usually called supremal convolution. A dual operation is the so called infimal convolution given by $(f \square g)(x)=\underset{y \in R^{d}}{\wedge} f(x-y)+g(y)$.

is closely related to the morphological erosion $\Theta$ because $f \Theta g=f \square(-\breve{g})$ where $\breve{g}$ is the reflection of g given by $\breve{g}(x)=g(-x)[8]$.Therefore $\oplus$ and $\square$ are supremal and infimal convolution, respectively, corresponding to the concept of a dilation and erosion operator on a lattice.

A mapping $\Delta$ which sends a signal $f$ to a transformed signal $\Delta(f)$ is called dilation translation invariant(DTI) system if it is a dilation, ie, $\Delta\left(\vee_{i} f_{i}\right)=\vee_{i}\left(\Delta f_{i}\right)$, and if it is translation- invariant, ie. $\Delta\left(f_{y}+c\right)=\Delta(f)_{y}+c$ for any shift y and any real constant $\mathrm{c}$.I $\mathrm{t}$ is easy to verify that a system is DTI if it is a horizontally shift-invariant and obeys the morphological supremum superposition principle $\Delta\left[\underset{i \in I}{\vee} f_{i}(x)+c_{i}\right]=\underset{i \in I}{\vee} \Delta\left(f_{i}(x)+c_{i}\right.$, where $\{$ fi $\}$ is $\quad$ any signal collection and $c_{i} \in R$.

Many important aspects of a DTI system can be determined in the time or spatial domain solely from knowledge of its output signal due to an elementary input signal, the morphological lower impulse $q_{\wedge}$ given by $q_{\wedge}(x):=\left\{\begin{array}{l}0, x=0 \\ -\infty, x \neq 0 .\end{array}\right.$

The corresponding output of the D T I system $\Delta$ when the input is the lower impulse is henceforth defined as its lower impulse response $: g:=\Delta\left(q_{\wedge}\right)$.

This uniquely characterizes a DTI system in the time domain,because any DTI system is equivalent to a supremal convolution(also called 'morphological dilation') by its lower impulse response: $\Delta(f)=f \oplus g$

Similarly ,a signal operator $\varepsilon: f \mapsto \varepsilon(f)$ is called an erosion translation invariant (ETI) system if it is horizontally shift -invariant and obeys the morphological infimum superposition principle $\varepsilon\left[\hat{}_{i \in i} f_{i}(x)+c_{i}\right]=\hat{\wedge_{i \in I}} \varepsilon\left(f_{i}\right)(x)+c_{i} \quad$ where $\quad c_{i} \in R$.If we define the upper impulse response h of an ETI system $\varepsilon$ as its response

$$
h:=\varepsilon(q \vee) \text { to the upper impulse } h=-\breve{g} \text {. }
$$

$q_{\vee}(x):=\left\{\begin{array}{l}0, x=0 \\ +\infty, x \neq 0 .\end{array}\right.$

then it follows that

$$
\varepsilon(f)=f \square h \text {. }
$$

When the ETI and DTI systems are related via an adjunction, then there is also a close relationship between their impulse 
responses. Namely, let $\varepsilon$ be an ETI system, and let $\Delta$ be its adjoint dilation. It is easy to show that $\Delta$ is a DTI system[11], and therefore $\Delta(f)=f \oplus g$, where $\mathrm{g}$ is the lower impulse response. The above relationship between the two theories inspired us for finding a generalized algebraic structure for Mathematical Morphology. Since it is the generalization ,no seperate proof is required for most of the results. However ,we tried to give proof for new propositions. Examples are given for some generalizations. We hope that this generalization is helpful for developing the theory of Mathematical Morphology. We also hope that this paper give the relation between combinatorial convexity, mathematical morphology and image processing.

\section{BASIC CONCEPTS}

\subsection{Definition: Moore family}

Moore family is defined by using a partially ordered set L .It also satisfies certain properties on $\mathrm{L}$.

Let $\mathrm{L}$ be a poset.

i) A subset $\mathrm{M}$ of $\mathrm{L}$ is a Moore family if every element of $\mathrm{L}$ has a least upper bound in $\mathrm{M}$.

[ $\forall x \in L, \exists y \in M, y \geq x$ and $\forall z \in M, z \geq x \Rightarrow z \geq y$ ]

ii) A closure operator on $\mathrm{L}$ is an increasing, extensive and idempotent operator from $\mathrm{L} \rightarrow$ L.[15]

\subsection{Proposition}

Let $\mathrm{L}$ be a poset. There is a one to one correspondence between Moore families in $\mathrm{L}$ and closings on $\mathrm{L}$, given as follows.

i) To a Moore family M, associate the closing defined by setting for every $x \in_{L} ;(x)$ is equal to the least $\mathrm{y} \in \mathrm{M}$ such that $\mathrm{y} \geq \mathrm{x}$. [15]

ii) To a closing, one associates the Moore family $\mathrm{M}$ which is the invariance domain of $\mathrm{M}=\mathrm{In}$ (i.e. $\mathrm{M}=\{\mathrm{O} / \in\}$.

\subsection{Convex Geometry}

Let $\mathrm{S}$ be a set ,consider the family $\mathrm{T}$ of subsets of $\mathrm{S}$ with the following properties:

$\varphi \in T_{,} S \in T_{\text {, }}$

\section{$A, B \in T$ implies $A \cap B \in T$}

This family defines a closure operator $\phi(X)=\bigcap\{A \in T, X \subseteq A\} \times[1]$

Every closure operator defines a family $T$ ' with the above properties. Elements of $\mathrm{T}$ or elements defined by $\phi$ will be called convex. We call the pair $(S, \phi)$ is a Convex geometry if $\phi$ verifies the anti-exchange axiom. [1] $\forall \mathrm{x}, \mathrm{y} \notin \phi(\mathrm{X}), x \neq y, x \in \phi(X \cup Y) \Rightarrow y \notin \phi(X)$.

In the same way,

If $\phi(X) \neq S, \exists p \in S \backslash \phi(X), \phi(X \cup p)=\phi(X) \cup p$

\subsection{Poset Geometry}

Corresponding to a partially ordered set, we have a graphical representation, known as Hasse Diagrams. So we can infer that Poset give some geometrical representation. In view of this we can define Poset Geometry.[1]

Let $\mathrm{P}$ be a partially ordered set and $\mathrm{X}$ be a subset of $\mathrm{P}$, Define $D_{p}(X)=\{y \in P, y \leq x$ for some $x \in X\},\left(\mathrm{P}, D_{p}\right)$ is a convex geometry called Poset geometry which are characterized by the following:

The convex geometry $(\mathrm{S}, \phi)$ arises from the poset geometry on a Poset $\mathrm{P}$ if and only if $\phi(A \cup B)=\phi(A) \cup \phi(B)$ $\forall A, B \subseteq S$.

\subsection{Dilation}

A dilation is defined by an operator $\delta: P(S) \rightarrow P(S)$ with the following properties: $\delta(\phi)=\phi, A, B \in P(S), \delta(A \cup B)=\delta(A) \cup \delta(B)$.

\subsection{Erosion}

An erosion is defined by an operator $\varepsilon: P(S) \rightarrow P(S)$ with the following properties:

$$
\varepsilon(S)=S, A, B \in P(S), \varepsilon(A \cap B)=\varepsilon(A) \cap \varepsilon(B)
$$

\subsection{Alexandroff space}

A topological space is an Alexandroff space if the intersection of any family of open sets is open(resp.the union of any family of closed sets is closed)

\subsection{Binary relation Canonically associated with Dilation}

Let $\delta$ be a dilation on S. For any dilation, define a binary relation as follows:

xRy is equivalent to $x \in \delta(y)$, for $x, y \in S$ or xR y is equivalent to $\delta(x) \subseteq \delta(y), \forall x, y \in S$. [1]

\subsection{Result}


Let $\delta$ be a dilation on S. R its binary relation canonically associated with it. Then the following are equivalent.

i. $\mathrm{R}$ is reflexive and transitive

ii. $\quad \mathrm{RRy}$ is equivalent to $\delta(x) \subseteq \delta(y)$

iii. $\delta$ defines a dual Moore family.

Proof:

$\mathrm{i} \Rightarrow$ ii .Since $\mathrm{R}$ is reflexive and transitive, $\delta(\mathrm{x}) \subseteq \delta(\mathrm{x})$ and $\delta(\mathrm{x}) \subseteq \delta(\mathrm{y})$. Therefore $\delta(x) \subseteq \delta(y)$

ii $\Rightarrow$ iii . Since $\delta(x) \subseteq \delta(y)$,by definition $\delta$ defines a dual Moore family.

iii $\Rightarrow$ i. Since $\delta$ defines a dual Moore family, it is both reflexive and transitive.

\subsection{Proposition}

Let $\mathrm{S}$ be a set . Let $\mathrm{N}: \mathrm{S} \rightarrow \mathrm{P}(\mathrm{S})$ corresponding to $\rho$ by

$\forall x, y \in S, x \in N(y) \Leftrightarrow y \in N(x) \Leftrightarrow x_{\rho} y$

and $\mathrm{N}(\mathrm{x})=\{y \in S / x \rho y\}$

Then (i) $\mathrm{N}$ separates $\mathrm{S}$ in a primary sense

(ii) $(\mathrm{S}, \mathrm{N})$ is a Poset geometry.

(iii) $(\mathrm{S}, \mathrm{N})$ is a To Alexandroff space.

\subsection{Proposition}

$(\mathrm{S}, \mathrm{N})$ is separated in a primary sense if $\mathrm{N}$ verifies the following two properties.

i. For any family $\left(\mathrm{x}_{\mathrm{i}}\right), \mathrm{i} \in \mathrm{I}$ of elements and for any element $\mathrm{x} \in S_{y}$ verifying $\mathrm{N}(\mathrm{x}) \subseteq \bigcup_{i \in I} N\left(x_{i}\right)$, $\exists j \in I$ such that $\mathrm{N}(\mathrm{x}) \subseteq \mathrm{N}\left(\mathrm{x}_{\mathrm{j}}\right)$

ii. $\quad \mathrm{N}(\mathrm{x})=\mathrm{N}(\mathrm{y})$ is equivalent to $\mathrm{x}=\mathrm{y}$ for any $\mathrm{x}, \mathrm{y} \in \mathrm{S}$

\subsection{Definition: Morphological Closure}

$\varphi=\operatorname{so\delta }$ is called a morphological closure and $\varphi(x)=\{y \in S / \delta(y) \subseteq \delta(x)\}$

\subsection{Result}

Let $\mathrm{S}$ be an infinite space and let $\mathrm{N}$ be defined by $\mathrm{R}$. Then $(\mathrm{S}, \mathrm{N})$ is a convex geometry if and only if $\forall X \subseteq S_{3}(S-N(x), \psi)$ is a To- Alexandroff space where
$\psi(\mathrm{A})=\mathrm{U}_{y \in A}[N(N(X) \cup y) \cap S-N(X)]$

\subsection{Proposition}

Let $\delta: P(v) \rightarrow P(w)$ and $\varepsilon: P(w) \rightarrow P(v)$ such that $N: v \rightarrow P(w)$ where $N(v)=\delta(\{v\}), \forall v \in V$ and $\delta=\delta_{\mathrm{N}}, \varepsilon=\varepsilon_{\mathrm{N}}$

Define $\delta_{N}(\mathrm{Y})=V-\varepsilon_{N}(W-Y), \forall Y \in P(W) \quad$ and $\varepsilon_{\mathbb{N}}(\mathrm{X})=\mathrm{W}-\delta_{\mathrm{N}}(\mathrm{V}-\mathrm{X}), \forall \mathrm{X} \in P(V)$.

Then $\delta_{\mathbb{N}}$ and $\varepsilon_{N}$ are dual by complementation of $\varepsilon_{\mathrm{N}}$ and $\delta_{\mathrm{N}}$.

Also $\varepsilon_{\mathbb{N}}$ is a dilation and $\delta_{\mathbb{N}}$ is an erosion. $\operatorname{Also}_{\mathbb{N}}=$ $\delta_{\rho}-1$ and $\varepsilon_{\mathbb{N}}=\varepsilon_{\rho}^{-1}$ where $\rho^{-1}$ is defined as $\mathrm{w} \rho^{-1}$ $v \Leftrightarrow v \rho w$ and $v \rho w \Rightarrow w \in \delta(v)=N(v)$.

\subsection{Proposition}

Let $\delta$ be a dilation and $\varepsilon$ an erosion. Let $\rho$ be the relation defined as before .Then

i. $\rho$ is reflexive and transitive.

ii. $v \rho w$ is equivalent to $\delta_{\mathrm{N}}(\mathrm{x}) \subseteq \delta_{\mathrm{N}}(\mathrm{y})$.

iii. $\delta_{\mathrm{N}}$ defines a Dual Moore family.

iv. $\varepsilon_{\mathrm{N}}$ defines a Moore family.

We can prove the above result using the definitions.

\section{GENERALIZED ALGEBRAIC STRUCTURE}

In this section we give general algebraic structure for Morphology and some definitions are restated using the generalization.

\subsection{Definition: Morphogenetic field}

Let $\mathrm{X} \neq \varphi$ and $\mathrm{W} \subseteq P(X)$ such that i) $\phi, \mathrm{X} \in \mathrm{W}$, ii) If $\mathrm{B}$ $\in \mathrm{W}$ then its complement $\bar{B} \in \mathrm{W}$ iii) If $\mathrm{B}_{\mathrm{i}} \in \mathrm{W}$ is a sequence of signals defined in $\mathrm{X}$, then $\bigcup_{n=1}^{\infty} B i \in \mathrm{W}$.

Let $\mathrm{A}=\{$

$\left.\phi: W \rightarrow U / \phi\left(\cup A_{i}\right)=\vee \phi\left(A_{i}\right) \& \phi\left(\wedge A_{i}\right)=\wedge \phi\left(A_{i}\right)\right\} \mathrm{T}$ hen $\mathrm{W}_{\mathrm{U}}$ is called Morphogenetic field where the family $\mathrm{W}_{\mathrm{u}}$ is the set of all image signals defined on the continuous or discrete image Plane $\mathrm{X}$ and taking values in a set $\mathrm{U}$. The pair $\left(\mathrm{W}_{\mathrm{u}}, \mathrm{A}\right)$ is 
called an operator space where $\mathrm{A}$ is the collection of operators defined on $\mathrm{X}$.

\subsection{Definition : Morphological space}

The triplet ( $\left.\mathrm{X}, \mathrm{W}_{\mathrm{u}}, \mathrm{A}\right)$ consisting of a set $\mathrm{X}$, a morphogenetic field $\mathrm{W}_{\mathrm{u}}$ and an operator $\mathrm{A}$ (or collection of operators) defined on $\mathrm{X}$ is called a Morphological space.

Note: If $\mathrm{X}=\mathrm{Z}^{2}$ then it is called Discrete Morphological space.

\subsection{Definition Convex Morphological space}

Let $\left(X, W_{u}, A\right)$ be a morphological space and $\left(W_{U}, A\right)$ be an operator space in $\left(X, W_{u}, A\right)$.

If $\mathrm{X}$ is a class of concave functions then $\left(X, W_{u}, A\right)$ is called concave morphological space. If $\mathrm{X}$ is a class of convex functions then $\left(X, W_{u}, A\right)$ is called convex morphological space.

\subsection{Proposition}

Every convex morphological space has * property.

\subsection{Definition * property}

Let $\left(X, W_{u}, A\right)$ be a morphological space and $\left(W_{U}, A\right)$ be an operator space in $\left(X, W_{u}, A\right)$.Let $x(\alpha) \in X$, then $x(\alpha)$ has at least one maxima or minima in $\mathrm{X}$.

\subsection{Proposition}

Every convex morphological space is optimizable.

\subsection{Definition: Geometrical Space}

Let $\left(X, W_{u}, A\right)$ be a morphological space and $\left(W_{U}, A\right)$ be an operator space in $\left(X, W_{u}, A\right)$.

If $\phi$ is an operator in $\mathrm{A}$ and in particular if $\phi$ satisfies (or defines a rule) in $W_{U}$ then the operator space $\left(W_{U}, \phi\right)$ is called a geometrical space and $\phi$ defines a morphological geometry in $W_{U}$.

\subsection{Proposition}

Let $\left(X, W_{u}, A\right)$ be a morphological space and $\left(W_{U}, A\right)$ be an operator space $\operatorname{in}\left(X, W_{u}, A\right)$.Suppose that $\varphi \in A, S \in W_{U} . X_{1}, X_{2} \in W_{U} \Rightarrow X_{1} \cap X_{2} \in W_{U}$ and $\phi(S)=\cap X_{1} \in W_{U} / S \subseteq X_{1} \quad$ then $\phi \quad$ defines a morphological geometry ,known as convex geometry if $\forall x, y \notin \phi(S), x \neq y, x \in \phi(S \cup Y) \Rightarrow y \notin \phi(S \cup x)$ . $\left(W_{U}, \phi\right)$ is called a convex geometrical space.
Also $\quad$ if $\quad \phi(S) \neq X$ then $\quad \exists z \in X-\phi(S)$ and $\phi(S \cup z)=\phi(S) \cup z$.

\subsection{Definition: Poset Geometry}

Let $(P, \leq)$ be a poset and $\mathrm{X}$ be a subset of $\mathrm{P}$.

Define $T_{p}(X)=y \in P / y \leq x$, for some $\quad x \in A$. Let $\left(X, W_{u}, A\right)$ be a morphological space and $W_{u}=P, A=T_{p}$. Then the operator space is $\left(P, T_{P}\right)$ defines a geometry known as poset geometry and $\left(P, T_{P}\right)$ is called a poset geometrical space.

\subsection{Proposition}

Let $\left(X, W_{u}, A\right)$ be a morphological space and let $\left(W_{U}, \phi\right)$ be a poset geometrical space in $\left(X, W_{u}, A\right)$. Then $\left(W_{U}, \phi\right)$ is called a convex geometrical space iff $\phi\left(X_{1} \cup X_{2}\right)=\phi\left(X_{1}\right) \cup \phi\left(X_{2}\right), \forall X_{1}, X_{2} \in X$.

\subsection{Proposition}

Let $\left(X, W_{u}, A\right)$ be a morphological space. Then for $\gamma(x) \in W_{U},(X, \gamma(x))$ is called an

anti matroid if $(X, \gamma(x))$ satisfies the following.

i) $\varphi \in \gamma(x), \gamma(x)$ is closed under union.

ii) For $S \in \gamma(x), S \neq \phi, \exists x \in S$ such that $S-x \in \gamma(x)$.

\subsection{Proposition(Adjunction)}

Let $\left(X, W_{u}, A\right) \&\left(Y, W_{u}, \bar{A}\right)$ be morphological spaces. The $\operatorname{pair}(A, \bar{A})$ is $\quad$ called an adjunction $\quad$ iff $A(X) \leq Y \Leftrightarrow X \leq \bar{A}(Y)$ where $\bar{A}$ is an inverse operator of A.

\subsection{Dilation and Erosion as Adjunctions}

Let $\left(X, W_{u}, \delta\right) \&\left(Y, W_{u}, \varepsilon\right)$ be a morphological spaces with operators dilation and erosion on A. Then $\delta(X) \leq Y \Leftrightarrow X \leq \varepsilon(Y)$.

\subsection{Proposition (For lattice)}

Let $\left(X, W_{u}, A\right) \&\left(Y, W_{u}, \bar{A}\right)$ be a morphological spaces. The pair $(A, \bar{A})$ is called an adjunction iff $\forall u, v \in X, \exists$ an 


$$
\begin{aligned}
& \text { adjunction } \quad\left(l_{u, v}, m_{v, u}\right) \text { on } \quad \mathrm{U} \quad \text { such that } \\
& \bar{A}(x(u))=\underset{v \in X}{\vee} m_{v, u}(x(v)) \text { and } \\
& A(y(v))=\hat{\wedge}_{u \in X} l_{u, v}(y(u)), \forall u, v \in X, x, y \in W_{U} .
\end{aligned}
$$

\subsection{Definition: Morphological Closure \& Morphological Kernel.}

The operator $\phi=\varepsilon \circ \delta$ defines a closure called morphological closure and $\phi^{*}=\delta \circ \varepsilon$ defines a kernel, called morphological kernel.

\subsection{Lemma}

Let $\left(X, W_{u}, A\right) \quad$ be a morphological space. $\phi^{*}(S)=\cup X_{1} \in W_{U} / X_{1} \subseteq S$ defines a kernel operator in A. The pair $\left(X, \phi^{*}(S)\right)$ is an anti matroid if $\phi^{*}$ satisfies the axiom: For $\phi^{*}(S) \neq \varphi, \exists z \in \phi^{*}(S), \phi^{*}(S-z)=\phi^{*}(S)-z$.

Proof:

Since $\phi^{*}(S) \in W_{U}$ where $W_{U}$ is a morphogenetic field in a morphological space $\left(X, W_{u}, A\right) \phi^{*}(S)$ is an anti matroid.

Direct proof.

Since $\phi^{*}(S-z) \subseteq S-z$, so $z \notin \phi^{*}(S-z)$.From monotonicity, $S-z \subseteq S \Rightarrow \phi^{*}(S-z) \subset \phi^{*}(S)$.There fore $\phi^{*}(S-z) \subset \phi^{*}(S)$

Conversely,

$\dot{\phi}^{*}(S)-z \subseteq S-z \Rightarrow \phi^{*}\left(\dot{\phi}^{*}(S)-z\right) \subseteq \phi^{*}(S-z)$.

Therefore $\left.\left.\phi^{*}(S)-z\right) \subset \phi^{*}(S-z) \Rightarrow \phi^{*}(S)-z\right)=\phi^{*}(S-z)$.

\subsection{Theorem}

Let $\left(X, W_{u}, A\right)$ be a morphological space. $(X, \phi)$ defines a convex geometry iff $\left(X, \phi^{*}\right)$ is an antimatroid.

\subsection{Seperation}

Let $\left(X, W_{u}, A\right),\left(X, W_{u}, \bar{A}\right)$ be morphological spaces. Let $(A, \bar{A})$ be adjunctions. $(X, A)$ is separated in a primary sense if A verifies the following two properties.
1) Let $x \in X, A(x) \subseteq \underset{\forall x_{i} \in W_{U}}{\cup} A\left(x_{i}\right) \Rightarrow \exists j \in I$ such that $A(x) \subseteq A\left(x_{j}\right)$

2) $A(x)=A(y) \Rightarrow x=y \forall x, y \in X$ and $\phi=A \circ \bar{A}$ defines a morphological closure.

\subsection{Theorem}

Let $\left(X, W_{u}, A\right)$ be a morphological space and $\phi=A \circ \bar{A}$ be the morphological closure. Then the following statements are equivalent.

1) A separates $X$ in a primary sense.

2) $\left(W_{U}, \phi\right)$ is a morphological geometrical space.

$\left(W_{U}, \phi\right)$ is a poset geometrical space.

\subsection{Theorem}

Let $\left(X, W_{u}, A\right)$ be a morphological space and let $\mathrm{X}$ be an infinite set and $\phi=A \circ A$ be the morphological closure. Then the following statements are equivalent.

1) A separates $X$ in a primary sense.

2) $\left(W_{U}, \phi\right)$ is a morphological geometrical space.

3) $\left(W_{U}, \phi\right)$ is a poset geometrical space

3) $\left(W_{U}, \phi\right)$ is a $\mathrm{T}_{0}$ Alexandroff space.

Proof:

3) $\Rightarrow 4)$

Let $\left(W_{U}, \phi\right)$ be a poset geometrical space. $\forall y \in \phi(y), \exists B \subseteq Y$,B being a finite set such that $y \in \phi(B) . \therefore y \in \phi(y) \Rightarrow y \in \phi(z)$ for $\quad$ some $\mathrm{z} \in \mathrm{Y} . \therefore \quad\left(W_{U}, \phi\right) \quad$ is $\quad$ an Alexandroff space $\because \forall x, y \in \phi(y)$, $x \neq y, x \in \phi(Y \cup y) \Rightarrow y \notin \phi(Y \cup x), \therefore$ is $\quad$ a $\quad \mathrm{T}_{0}$ space.

4) $\Rightarrow 1)$

Let $\left(W_{U}, \phi\right)$ is a $\mathrm{T}_{0}$ Alexandroff space. $\because$

$\phi=A \circ \bar{A}$,

$\phi(Y)=\{y \in X / \bar{A}(y) \subseteq \bar{A}(Y)\}$.

$\therefore \forall x \neq y, \bar{A}$ seperates $\mathrm{X}$ in a primary sense. 
1) $\Rightarrow 2)$

Let $\bar{A}$ separates $\mathrm{X}$ in a primary sense .Since $\bar{A}(x) \subseteq \bar{A}(y)$

$\phi(y)=A \circ \bar{A}(y)$ and

$\phi(y)=\{y \in X / \bar{A}(Y) \subseteq \bar{A}(y)\}$.

$\phi(Y)=\cap Y_{\overline{1}} \in W_{U} / Y \subseteq Y_{1}$

$\Rightarrow y \in X / y R x \forall x \in Y$

$\therefore\left(W_{U}, \phi\right)$ is a morphological geometrical space.

2) $\Rightarrow 3)$

Let $\left(W_{U}, \phi\right)$ is a morphological geometrical space. $\Rightarrow \phi(Y)$ is an ideal of $\mathrm{X}$. $\Rightarrow \phi(Y)$ is closed and $\mathrm{R}$ is an order relation. $\therefore\left(W_{U}, \phi\right)$ is a poset geometrical space.

Hence the result.

\section{ACKNOWLEDGMENTS}

The first author thank management of Rajagiri School of Engineering \& Technology for their support in publishing this research paper.

\section{REFERENCES}

[1] Alain Bretto, Enzo Maria Li Marzi. Mathematical Morphology and Poset Geometry.

[2] Alexandre X. Falca o, Jorge Stolfi, and Roberto de Alencar Lotufo The Image Foresting Transform: Theory, Algorithms, and Applications, IEEE Transactions on Pattern Analysis and Machine Intelligence, Vol 26,No.1 January 2004.

[3] Chu-Song Chen, Yi-Ping Hung, Theoretical Aspects of Vertically Invariant Gray-Level Morphological Operators and Their Application on Adaptive Signal and Image Filtering, IEEE transactions on signal processing, vol. 47, no. 4, april 19991049

[4] Frank Y .Shih ,Image Processing and Mathematical Morphology, CRC Press,2009.

[5] G. CROMBEZ, The Matheron Representation Theorem for Gray Scale Morphological Operators, Proceedings of the American Mathematical Society Volume 108, Number 3, March 1990.

[6] Henk J.A.M Heijmans ,John Goustias Mathematical Morphology, I.O.S Press.

[7] [H.J.A.M Heijmans, Morphological Image Operators, Boston, M.A Academic, 1994 .
[8] Henk J.A.M Heijmans \& Petros Maragos, Lattice Calculus of the Morphological Slope Transforms, IEEE Transactions on Image Processing,22-1994,601-625.

[9] Heijmans, H.J.A.M , and Ronse,C.The algebraic basis of Mathematical Morphology -Part I, Dilations and Erosions, Computer vision, Graphics and Image Processing,50(1990) 245-295.

[10] Henk Heijmans, Rein Van Den Boomgaard ,Morphological scale space- operators.

[11] Javier Vidal\& Jos'e Crespo, Sets Matching in Binary Images Using Mathematical Morphology , International Conference of the Chilean Computer Science Society.

[12] Jean Cousty, Laurent Najman and Jean Serra, Some morphological operators in graph spaces, ISSM-2009.

[13] J .Serra, Image Analysis and Mathematical Morphology, New York Academic 1982.

[14] Matheron. G ,Random Sets and Integral Geometry, John Wiley and Sons, New York, 1975.

[15] Nathalie Caspard and Bernard Monjardet, The lattices of Moore families and closure operators on a finite set: A survey, CERMSEM, Maison des Sciences Économiques (Université Paris1), 106-112, bd de l' Hô̆ pital, 75013, Paris, France.

[16] P .Maragos and R.W Schafer, "Morphological system for multi dimensional signal processing" Proc.IEEE,Vol, 78,P.D 690-710,April 1990.

[17] Petros Maragos,Morphological Skeleton representation and coding of Binary images.IEEE -Transactions on Acoustics, Speech and Signal Processing, 34 -1986, 1228-1244.

[18] Petros Maragos,Pattern spectrum and multi scale shape representation. IEEE Transactions on Pattern analysis and machine intelligence,11,(1989),701-716.

[19] P.Maragos,Slope Transforms:Theory and Applications to Non Linear Signal Processing,IEEE Transactions on Signal Processing,Vol.43, No. 4,April 1995.

[20] P. Radhakrishnan, B. S. D. Sagar, and B. Venkatesh, Morphological Image Analysis of Transmission Systems, Transactions on Power Delivery, vol.20, no.1, january 2005 219 